\section{OP44 FEEDING DIFFICULTIES AND MATERNAL CONCERNS AT AGE 3 YEARS ARE ASSOCIATED WITH A DECLINE IN CHILDREN'S DIET QUALITY TO AGE 6}

${ }^{1} \mathrm{M}$ Jarman*, ${ }^{2,3} \mathrm{SM}$ Robinson, ${ }^{2,3} \mathrm{WT}$ Lawrence, ${ }^{2,3} \mathrm{ME}$ Barker, ${ }^{2,3} \mathrm{KM}$ Godfrey, ${ }^{2,3} \mathrm{C}$ Cooper, ${ }^{2,3} \mathrm{HM}$ Inskip, ${ }^{2} \mathrm{~J}$ Baird. ${ }^{1}$ Agriculture, Food and Nutrition Science, University of Alberta, Edmonton, Canada; ${ }^{2}$ MRC Lifecourse Epidemiology Unit, University of Southampton, Southampton, UK; ${ }^{3}$ NIHR Southampton Biomedical Research Centre, University of Southampton, Southampton, UK

10.1136/jech-2017-SSMAbstracts.44

Background Cross-sectional studies show that picky eating behaviours in young children are associated with poorer quality diets. Limited longitudinal research explores whether these effects persist until later childhood. We assessed feeding difficulties and eating behaviours at age 3 years and their influence on quality of diet at age 3 and 6 .

Methods The Southampton Women's Survey is a prospective cohort study of women pre-pregnancy, those that became pregnant and their children. Complete data for this analysis were available on 1946 mother-child pairs. When children were age 3 years, mothers completed a questionnaire assessing frequency of feeding difficulties, and concerns about specific eating behaviours in three categories indicating the level of the problem. Diet was assessed using a food frequency questionnaire. Principal components analysis was used to examine dietary patterns; a z-score was derived with higher scores reflecting a better quality of diet. Dietary assessment was repeated at age 6 years. Linear regression models were used to assess associations between feeding difficulties, concerns about eating behaviours and diet scores at age 3 and 6. Models were adjusted for maternal age, education and number of children, and interactions between feeding difficulties and maternal concern about eating behaviours were assessed.

Results At age 3 more frequent feeding difficulties were associated with poorer quality diets $[\beta-0.14 \mathrm{SD} /$ category $(95 \% \mathrm{CI}$ $-0.18,-0.09)$ ]. Greater maternal concern about the eating behaviours 'my child refused to eat the right foods' and 'my child has been choosy with food' was associated with poorer quality diet at age $3[\beta-0.33 \mathrm{SD} /$ category $(95 \% \mathrm{CI}-0.43$,0.23 ) and $\beta-0.20 \mathrm{SD} /$ category $(95 \% \mathrm{CI}-0.31,-0.09)$, respectively] compared to children whose mothers were less concerned. These associations remained at age 6, even after adjustment for diet score at age 3, suggesting that greater maternal concern about their child 'refusing to eat the right foods' and 'being choosy with food' was associated with a worsening of children's diet quality to age $6[\beta-0.13 \mathrm{SD} /$ category $(95 \% \mathrm{CI}-0.23,-0.04)$ and $\beta-0.16 \mathrm{SD} /$ category (95\% CI $-0.27,-0.05)$, respectively].

Conclusion These findings suggest that the way mothers respond to their child's eating behaviours at age 3 might influence a decline in children's quality of diet until age 6 . Heightened maternal concern may generate a negative feeding environment which exacerbates picky eating behaviours in young children. Family support during preschool years for managing eating behaviours could contribute to more optimal diets in early childhood.

\section{Behaviours 1}

\section{OP45 THE EFFECT OF MULTIPLE RISK BEHAVIOURS IN ADOLESCENCE ON EDUCATIONAL ATTAINMENT AT AGE 16 YEARS: A UK BIRTH COHORT STUDY}

CL Wright*, RR Kipping, M Hickman, J Heron, R Campbell. School of Social and Community Medicine, University of Bristol, Bristol, UK

10.1136/jech-2017-SSMAbstracts.45

Background Single health risk behaviours are negatively associated with educational outcomes, but any relationship between multipe risk behaviours (MRB) and educational attainment has not been explored. We assessed the extent to which simultaneous engagement in MRB during adolescence is associated with examination performance at age 16 years.

Methods Data from the Avon Longitudinal Study of Parents and Children (ALSPAC), a population based birth cohort study of children born in England between 1991 and 1992 were used in linear and logistic regression analyses. We estimated the association between the total number of a wide range of health risk behaviours (including tobacco smoking, hazardous alcohol use and TV viewing) ranging from 0 to 13 behaviours, and educational attainment. The outcomes were: capped General Certificate of Secondary Education (GCSE) score and odds of attaining five or more GCSEs at grades A*-C. Multiple imputation was used to account for missing data.

Results We found that engagement in MRB was strongly associated with poorer educational attainment and each additional risk equated to a one grade reduction in capped GCSE score or a reduced odds of attaining five or more $\mathrm{A}^{*} \mathrm{C}$ grades of $23 \%$. The average cohort member engaged in $3 \mathrm{MRB}$ and would therefore have an associated reduction in their GCSE examinations equivalent to three grades in one examination, or reduced odds of attaining five or more $\mathrm{A} * \mathrm{C}$ grades of $69 \%$.

Conclusion Our findings show for the first time that engagement in a greater number of MRB during adolescence is strongly associated with poorer educational attainment at age 16 years. Preventing MRB could improve educational attainment and thereby directly and indirectly improve longer term health outcomes.

\section{OP46 CHANGES IN CONSUMPTION OF ADDED SUGARS FROM AGE 13 TO 30 YEARS: A SYSTEMATIC REVIEW AND META-ANALYSIS OF LONGITUDINAL STUDIES}

EM Winpenny*, TL Penney, K Corder, M White, EM F van Sluijs. MRC Epidemiology Unit, University of Cambridge, Cambridge, UK

\subsection{6/jech-2017-SSMAbstracts.46}

Background Intake of added sugar among adolescents is generally above recommended levels. Added sugar intake has been associated with weight gain and cardiometabolic risk factors during adolescence. Dietary habits developed during this 
period may persist into adulthood, increasing chronic disease risk in later life. A better understanding of the underlying trajectories of sugar consumption across adolescence and early adulthood will help inform appropriate interventions for this age group. This systematic review investigated changes in intake of added sugars and sugary foods and drinks, and determinants of such changes, between the ages of 13 and 30 years.

Methods In accordance with the registered protocol (PROSPERO: CRD42015030126), 7 electronic databases were searched in January 2016 for longitudinal studies of diet during adolescence or early adulthood. The papers retrieved were screened for studies including multiple measures of intake of sugars or sugary foods or drinks in cohort participants between the ages of 13 and 30 years. Data from included studies were extracted and analysed using random-effects meta-analysis, by the three main nutrient and food group categories identified.

Results We identified 23 papers reporting longitudinal data on intake of added sugar or sucrose $(n=5)$, sugar-sweetened beverages $(\mathrm{SSBs})(\mathrm{n}=20)$ and/or confectionery $(n=9)$. Eight papers reported data from the US, with Norway, Sweden and Australia also contributing several papers. On average, we found a per-year of age decrease in added sugar or sucrose intake $(-0.28 \%$ total energy intake $(95 \%$ CI $-0.44 ;-0.12))$, a decrease in confectionery consumption $(-0.20$ servings/week $(95 \%$ CI $-0.41 ;-0.001)$ ) and a non-significant decrease in SSB consumption (-0.15 servings/week (95\% CI -0.32 ; 0.02)). A small number of studies reported associations between behavioural determinants (e.g. screen time and fast food consumption) and change in intake of added sugar. Only three papers reported data beyond the age of 21 which weakens applicability of these findings to the early adulthood years.

Conclusion This review demonstrates a decrease in added sugar intake from adolescence to early adulthood, which may suggest an opportunity to capitalise on such changing preferences with interventions to further improve dietary choices within this age range. Improved longitudinal data is needed to further develop our understanding of changes in added sugar consumption into early adulthood and determinants of these changes.

\section{OP47 RELATIONSHIPS BETWEEN SUGAR INTAKE FROM SWEET FOOD AND BEVERAGES, COMMON MENTAL DISORDER AND DEPRESSION: PROSPECTIVE FINDINGS FROM THE WHITEHALL II COHORT STUDY}

A Knüppel*, MJ Shipley, CH Llewellyn, EJ Brunner. Department of Epidemiology and Public Health, University College London, London, UK

\subsection{6/jech-2017-SSMAbstracts.47}

Background In the face of high rates of mood disorders and an overconsumption of sugar, intake of sweet food, beverages and added sugars has been linked with depressive symptoms in several populations. It remains unclear if this association is causal or due to reverse causation (the influence of mood on sugar intake). Our study aimed to investigate systematically cross-sectional and prospective associations between sugar intake from sweet food/beverages, common mental disorder (CMD) and depression, and to examine the role of reverse causation as potential explanation for the observed linkage.
Methods We analysed repeated measures (23 245 person-observations) from the Whitehall II cohort study. The study recruited non-industrial civil servants from 1985 to 1988 . The most recent data used in this analysis was collected in 2013. CMD was measured with the 30-item General Health Questionnaire and depression with the 20-item Centre for Epidemiologic Studies Depression scale. Sugar intake from sweet food/beverages was assessed using food frequency questionnaires. Prospective analyses included 2, 5 and 10 year followup periods. We modelled associations using random effects regression using Stata 14.

Results Cross-sectional analyses showed positive associations. In prospective analyses we found a positive association of sugar intake from sweet food/beverages with incident CMD in men and with recurrent depression in women. Men in the highest tertile of intake had a 24\% increased odds of incident CMD after 5 years (95\% CI: 1.02, 1.48) independent of health behaviours, socio-demographic and diet-related factors, adiposity and other diseases (fully adjusted model). Women had a $36 \%$ increased odds for recurrent depression per $30 \mathrm{~g}$ increment $(95 \% \mathrm{CI}: 1.03,1.80)$ in fully adjusted models, associations using tertiles of sugar intake from sweet food/beverages were similar but not statistically significant when adjusted for diet-related factors. Notably, neither CMD nor depression predicted changes in sugar intake from sweet food/beverages. Conclusion Our findings using repeated measures follow-up data over 22 years suggest an adverse effect of sugar intake from sweet food/beverages on long-term psychological health. These results add further support for public health interventions which promote reduced sugar intake to improve overall health.

\section{OP48 AGE, SEX AND SOCIOECONOMIC INEQUALITIES IN FRUIT AND VEGETABLE INTAKE IN UK ADULTS, 1986- 2012}

A Yau*, J Adams, P Monsivais. Centre for Diet and Activity Research, MRC Epidemiology Unit, University of Cambridge, Cambridge, UK

\subsection{6/jech-2017-SSMAbstracts.48}

Background Low fruit and vegetable (FV) consumption is associated with cardiovascular disease and some cancers. The UK recommendation is that adults should consume at least $400 \mathrm{~g}$ $(5 \times 80 \mathrm{~g}$ portions) of $\mathrm{FV}$ per day. However, the most recent available data indicate that over $80 \%$ of UK adults do not adhere to the '5-a-day' recommendation. This study investigates whether the level of adherence has changed over time. Further, we look at number of FV portions consumed by age, sex and socioeconomic position across the three time points between 1986 and 2012.

Methods We used three surveys for this repeated cross-sectional analysis: the National Diet and Nutrition Survey (2000/ 01 and 2008/12) and a similar survey from 1986/87, the Dietary and Nutritional Survey for British Adults. These surveys employ multistage random sampling. FV intake was assessed using food diaries and demographic information collected by an interviewer. We included adults aged 19 to 64, who adequately completed a food diary, which gave sample sizes of 2047, 1724 and 1512, in 1986/87, 2000/01 and 2008/12 respectively. To test for socioeconomic inequalities, respondents were categorised into four groups (professional/managerial (I/ II); skilled non-manual (IIINM), skilled manual (IIIM); partly 